\title{
Critical analysis of the potential for therapeutic targeting of mammalian target of rapamycin (mTOR) in gastric cancer
}

This article was published in the following Dove Press journal:

Gastrointestinal Cancer:Targets and Therapy

8 April 2014

Number of times this article has been viewed

\author{
Mikito Inokuchi' \\ Keiji Kato' \\ Kazuyuki Kojima ${ }^{2}$ \\ Kenichi Sugihara' \\ 'Department of Surgical Oncology, \\ ${ }^{2}$ Department of Minimally Invasive \\ Surgery, Tokyo Medical and Dental \\ University, Tokyo, Japan
}

Correspondence: Mikito Inokuchi Department of Surgical Oncology, Tokyo Medical and Dental University, I-5-45, Yushima, Bunkyo-ku,

Tokyo II 3-85 I9, Japan

Tel +8I 35803526 I

Fax $+8 \mid 358030139$

Email m-inokuchi.srg2@tmd.ac.jp

\begin{abstract}
Multidisciplinary treatment including chemotherapy has become the global standard of care for patients with metastatic gastric cancer (mGC); nonetheless, survival remains poor. Although many molecular-targeted therapies have been developed for various cancers, only anti-HER2 treatment has produced promising results in patients with $\mathrm{mGC}$. Mammalian target of rapamycin (mTOR) plays a key role in cell proliferation, antiapoptosis, and metastasis in signaling pathways from the tyrosine kinase receptor, and its activation has been demonstrated in gastric cancer (GC) cells. This review discusses the clinical relevance of mTOR in GC and examines its potential as a therapeutic target in patients with $\mathrm{mGC}$. Preclinical studies in animal models suggest that suppression of the mTOR pathway inhibits the proliferation of GC cells and delays tumor progression. The mTOR inhibitor everolimus has been evaluated as second- or third-line treatment in clinical trials. Adverse events were well tolerated although the effectiveness of everolimus alone was limited. Everolimus is now being evaluated in combination with chemotherapy in Phase III clinical studies in this subgroup of patients. Two Phase III studies include exploratory biomarker research designed to evaluate the predictive value of the expression or mutation of molecules related to the Akt/mTOR signaling pathway. These biomarker studies may lead to the realization of targeted therapy for selected patients with $\mathrm{mGC}$ in the future.
\end{abstract}

Keywords: gastric cancer, mTOR, everolimus

\section{Introduction}

Gastric cancer (GC) is the fourth most common cancer, with 989,600 newly diagnosed cases worldwide in 2008 , accounting for about $8 \%$ of all newly diagnosed cancers. ${ }^{1}$ GC is the second leading cause of cancer-related mortality, with 738,000 deaths per year. The outcomes of GC remain poor, with an estimated relative 5-year survival rate of $25 \%$ in Europe. ${ }^{2}$ In contrast, the 5-year survival rate was $68.9 \%$ in a study of 13,626 patients treated in Japan in $2002 .^{3}$ The higher survival rate in Japan is attributed to early detection of GC by cancer screening programs and aggressive dissection of regional lymph nodes by advanced surgical techniques. At present, the treatment of choice for advanced GC is complete surgical removal of the tumor and adjacent lymph nodes. Nonetheless, many patients with advanced disease have recurrence, probably caused by the presence of occult micrometastasis unable to be managed by surgery alone. Multidisciplinary treatment strategies including surgery with chemotherapy or radiotherapy prolong survival; however, the benefits of therapeutic approaches such as chemotherapy and radiotherapy remain very limited in far advanced GC. 


\section{Current chemotherapy with cytotoxic agents for GC}

Median overall survival time (MST) was 10 to 13 months in patients with metastatic or unresectable gastric cancer (mGC) who received chemotherapy including multiple cytotoxic agents. ${ }^{4-6} \mathrm{~S}-1$ is an oral fluoropyrimidine preparation consisting of tegafur, 5-chloro-2,4-dihydropyrimidine, and potassium oxonate. The results of three Phase III studies comparing S-1based combination chemotherapy with S-1 monotherapy in Japan have suggested that cisplatin is the best partner for S-1. In the SPIRITS study, chemotherapy-naive patients with mGC were randomly assigned to receive either S-1 plus cisplatin or S-1 alone. ${ }^{4}$ The MST was significantly longer in the S-1 plus cisplatin group than in the $\mathrm{S}-1$ alone group (13.0 versus 11.0 months, $P=0.04)$. The TOP-002 study was conducted to compare the effectiveness of S-1 plus irinotecan with that of S-1 alone in patients with previously untreated advanced GC. ${ }^{5}$ The S-1 and irinotecan combination was not significantly superior to S-1 alone in terms of overall survival, but it was associated with longer MST than S-1 alone (12.8 versus 10.5 months, $P=0.23$ ). The START study compared S-1 plus docetaxel with S-1 alone in patients with mGC. ${ }^{6} \mathrm{~S}-1$ plus docetaxel was shown to be significantly superior to S-1 alone (MST, 12.5 versus 10.8 months, $P=0.04$ ). The FLAGS trial was conducted in Western countries, and patients with mGC were assigned to receive either cisplatin plus S-1 or cisplatin plus infusional fluorouracil. ${ }^{7}$ In Japan, S-1 has been administered in a daily dose of $80 \mathrm{mg} / \mathrm{m}^{2}$ of body surface area in many clinical trials. However, a lower daily dose of $50 \mathrm{mg} / \mathrm{m}^{2}$ was used in the FLAGS study because diarrhea occurred as dose-limiting toxicity in initial clinical trials performed in Western countries. ${ }^{8}$ In the FLAGS study, overall survival did not differ significantly between the patients who received cisplatin plus S-1 and those who received cisplatin plus infusional fluorouracil ( 8.6 versus 7.9 months, $P=0.20$ ); however, cisplatin plus S-1 had significant safety advantages in terms of treatment-related deaths and adverse events such as neutropenia, complicated neutropenia, and stomatitis. S- 1 is therefore considered an alternative to infusional fluorouracil, even in Western countries.

Capecitabine is another oral fluoropyrimidine that is activated in tumor tissue by a three-step enzymatic conversion, the final step of which requires thymidine phosphorylase. The REAL2 trial was designed to determine whether fluorouracil could be replaced by capecitabine and cisplatin be replaced by oxaliplatin in the triplet regimen of epirubicin, cisplatin, and infused fluorouracil, which had been widely used to treat mGC in Europe. ${ }^{9}$ The MST of the patients who received combined chemotherapy with epirubicin, oxaliplatin, and capecitabine (EOC) was significantly longer than that of the patients who received epirubicin, cisplatin, and infused fluorouracil (11.3 versus 9.9 months, $P=0.02$ ).

Triplet regimens including docetaxel have also been developed. The V325 trial compared the therapeutic usefulness of docetaxel, cisplatin, and fluorouracil (DCF) with that of cisplatin and fluorouracil (CF) in the Phase III part of the study in patients with mGC. ${ }^{10}$ The MST was significantly longer in the DCF group than in the CF group (9.2 versus 8.6 months, $P=0.02)$. However, $\mathrm{DCF}$ was more frequently associated with toxic effects such as febrile neutropenia, severe diarrhea, and severe neurosensory impairment. Because these regimens were poorly tolerated clinically and required modification, a triplet regimen of docetaxel, cisplatin, and S-1 was developed and used to treat $\mathrm{mGC}$ in a Japanese Phase III trial. ${ }^{11}$

\section{Receptor tyrosine kinase-targeting agents for $\mathbf{G C}$}

Activated receptor tyrosine kinase (RTK) regulates many key signaling pathways participating in cell growth, survival, organ morphogenesis, vascularization, and tissue repair and regeneration. ${ }^{12}$ RTK activity is strictly regulated in normal cells, whereas dysregulation or constitutive activation of RTK has been found in various cancers. Deregulated activation of RTK signaling can be caused by gene mutation, gene amplification, and overexpression of both receptor and ligand, which has been shown to correlate with the progression of various human cancers. In GC, high gene or protein expression of many RTKs including epidermal growth factor receptor (EGFR) ${ }^{13}$ human epidermal growth factor receptor 2 (HER2), ${ }^{14,15}$ vascular endothelial growth factor receptor (VEGFR), ${ }^{16,17}$ and c-mesenchymal-epithelial transition (c-MET) $)^{18,19}$ correlate with tumor progression or patient survival. Since various RTKs are involved in many aspects of tumor progression, these enzymes are considered promising therapeutic targets. Agents targeting RTK signaling pathways include antagonists of the ligands binding RTKs, inhibitors of receptor activation such as monoclonal antibodies, tyrosine kinase inhibitors (TKI), and inhibitors of downstream pathways.

Trastuzumab, an anti-HER2 monoclonal antibody, was the first RTK-targeting agent approved for the indication of GC worldwide. In the ToGA study, a Phase III trial performed in patients with advanced GC, the MST was significantly longer in patients who received trastuzumab plus chemotherapy (cisplatin and fluorouracil or capecitabine) than in patients who received chemotherapy alone 
(13.8 versus 11.1 months, $P=0.0046) .{ }^{20}$ Eligible patients in the ToGA study had advanced GC with either strongly positive (3+) HER2 expression on immunohistochemical (IHC) analysis or positive HER2 expression on fluorescence in situ hybridization. An exploratory analysis according to HER2 status suggested that overall survival was longer in patients with high expression of HER2 protein than in patients with low expression (16.0 versus 11.8 months, $P=0.0002$ ).

Two agents targeting EGFR, a member of the HER family along with HER2, provided no additional benefits as compared with chemotherapy alone in Phase III clinical trials. In the EXPAND trial evaluating the efficacy of cetuximab, a monoclonal antibody of EGFR, the MST of patients assigned to cetuximab plus chemotherapy (capecitabine and cisplatin) was similar to that of patients assigned to chemotherapy alone (9.4 versus 10.7 months, $P=0.95) .{ }^{21}$ In the REAL3 study of panitumumab, another monoclonal antibody of EGFR, the MST of patients who received panitumumab plus modified-dose EOC was significantly inferior to that of patients who received EOC alone (8.8 versus 11.3 months, $P=0.013$ ); however, the lower doses of cytotoxic agents in the panitumumab plus modified-dose EOC group might have diminished effectiveness. ${ }^{22}$ No biomarker was identified, and $K R A S$ or $B R A F$ mutations, which are associated with resistance to anti-EGFR treatment in colon cancer, were rarely found in that study. Lapatinib, a dual TKI against EGFR and HER2, also did not provide any additional benefit as second-line chemotherapy in patients with $\mathrm{mGC} .^{23}$

Bevacizumab is a monoclonal antibody targeting VEGF-A, which activates VEGFR-1 and VEGFR-2. In the AVAGAST trial, a Phase III study in patients with $\mathrm{mGC}$, the MST of patients assigned to bevacizumab plus toxic chemotherapy (fluoropyrimidine plus cisplatin) was not significantly superior to that of patients assigned to toxic chemotherapy alone ( 12.1 versus 10.1 months, $P=0.10$ ), although progression-free survival (PFS) was significantly prolonged by concurrent treatment with bevacizumab (6.7 versus 5.3 months, $P=0.004) .{ }^{24}$ In the biomarker evaluation substudy of the AVAGAST trial, high plasma vascular endothelial growth factor (VEGF)-A levels and low tumor neuropilin-1 expression were associated with trends toward improved overall survival, but only in non-Asian patients. ${ }^{25}$ Ramucirumab is a monoclonal antibody targeting the extracellular domain of VEGFR-2. In the REGARD study, a Phase III trial of second-line chemotherapy for $\mathrm{mGC}$, ramucirumab prolonged overall survival as compared with best supportive care (MST 5.2 versus 3.8 months, $P=0.047) .{ }^{26}$
Rilotumumab is a monoclonal antibody designed to inhibit the hepatocyte growth factor (HGF) binding c-MET pathway. In a Phase II study in $\mathrm{mGC}$, the additive effect of rilotumumab was clinically significant among patients with high c-MET expression. ${ }^{27}$ In that study, c-MET expression did not frequently overlap with HER2 status, suggesting that c-MET inhibitors can be effective against tumors without HER2 expression.

Foretinib, a multikinase inhibitor of c-MET, VEGFR2, and three other receptors, lacked efficacy in Phase II studies of patients with mGC. ${ }^{28}$ Sorafenib is an oral multitargeted TKI that inhibits VEGFR-1, VEGFR-2, VEGFR-3, platelet derived growth factor receptor (PDGFR), B-Raf, Raf-1, and c-Kit. A Phase II study of sorafenib combined with docetaxel and cisplatin resulted in survival similar to that obtained in other studies of chemotherapy alone. ${ }^{29}$ Sunitinib is also a multitargeted TKI targeting rearranged during transfection (RET), VEGFR-1, VEGFR-2, VEGFR-3, PDGFR $\alpha$, PDGFR $\beta$, FMS-like tyrosine kinase-3 (Flt3), c-KIT, and colony-stimulating factor receptor. Sunitinib has been evaluated as monotherapy in two Phase II studies but was not effective as second-line treatment for mGC. ${ }^{30,31}$

\section{Introduction to mammalian target of rapamycin}

Mammalian target of rapamycin (mTOR) has been recognized as a key regulator of cell growth, proliferation, metabolism, and angiogenesis. ${ }^{32-34}$ mTOR functions by integrating extracellular signals, such as growth factors and hormones, with amino acid availability and intracellular energy status to control translation rates and additional metabolic processes. ${ }^{35}$ Abnormal mTOR signaling has been associated with numerous pathological conditions, including cancer, immune disorders, diabetes mellitus, and cardiovascular and neurological diseases. ${ }^{36}$

mTOR exists as part of two functionally distinct protein complexes, mTORC1 and mTORC2. mTORC1 consists of mTOR catalytic subunit and two other proteins, regulatoryassociated protein of $\mathrm{mTOR}$ (raptor) and mammalian lethal with SEC13 protein 8 (mLST8), the latter of which is also known as $\mathrm{G}$ protein beta subunit-like. ${ }^{37-39}$ Raptor might have roles in mTORC1 assembly, recruiting substrates to mTORC1, and in regulating mTORC1 activity. ${ }^{40,41}$ The strength of the association between MTOR and raptor is regulated by nutrients and other signals that control the mTORC1 pathway. Signaling from growth factors is mediated to mTORC1 via the $\mathrm{PI} 3 \mathrm{~K} / \mathrm{Akt}$ pathway. The tuberous sclerosis complex (TSC1 and TSC2) is degraded by Akt, permitting mTORC1 activation. $\mathrm{mTORC} 1$ promotes protein synthesis via downstream 
effectors, such as the regulators of ribosomal S6 kinase 1 (S6K1) and eukaryotic translation initiation factor 4E binding protein 1 (4E-BP1). ${ }^{42,43}$ mTORC1-activated S6K1 promotes ribosomal protein translation and is an important regulator of cell size. 4E-BP1 is inactivated by $\mathrm{mTORC} 1$, and release of eIF4E from 4E-BP1 enables the formation of the active eIF4F complex, which is required for cap-dependent translation of mRNA. mTORC2 contains mTOR and mLST8 as well as two other proteins, rapamycin-independent companion of mTOR (rictor) and $\mathrm{mSin} 1$ (also known as mitogen-activated proteinkinase-associated protein), instead of raptor. ${ }^{44,45}$ Both rictor and $\mathrm{mSin} 1$ are necessary for phosphorylation of Akt, and then mTORC2 activates the positive feedback loop. Unlike raptor, the interaction between rictor and mTOR does not seem to be regulated by upstream signals. On the other hand, mTORC1-mediated S6K1 activation can inhibit mTORC2 through a negative feedback loop, thereby suppressing Akt activation. ${ }^{46,47}$ mTORC1 inhibits uncoordinated 51-like kinase 1 (ULK1) and ULK2, linked to starvation-induced autophagy. mTORC1 interrupts the binding of mammalian autophagy-related protein 13 to ULK, thereby inhibiting the phosphorylation of the focal adhesion kinase-family interacting protein, FIP200, and inducing autophagy. ${ }^{48}$

The location of mTOR may have an important role in regulation of its signaling pathway. A nuclear transport signal in mTOR is critical for its downstream cytoplasmic signaling to $\mathrm{S} 6 \mathrm{~K} 1 .{ }^{49}$ The rictor and $\mathrm{mSin} 1$ components of mTORC2 were translocated from the nucleus to the cytoplasm by treatment with rapamycin, an mTOR inhibitor, in human fibroblasts. ${ }^{50}$

\section{Clinical significance of $m$ TOR expression in GC}

We summarize the expression of phosphorylated mTOR (p-mTOR) or mTOR in GC on IHC analysis and clinical outcomes in Table 1. We previously reported that the localization of p-mTOR on IHC analysis is differentially related to tumor progression and patient survival in GC. ${ }^{51}$ The cytoplasmic expression of p-mTOR in GC cells was significantly associated with tumor depth, lymph-node metastasis, tumor stage, and poor survival while the nuclear expression of $\mathrm{p}-\mathrm{mTOR}$ was significantly associated with favorable survival. Similar results were obtained in several studies. The expression of p-mTOR on IHC analysis was significantly associated with lymph-node metastasis and tumor stage in a larger study, and p-mTOR detected in cytoplasm or cell membrane was an independent prognostic factor. ${ }^{52}$ However, overexpression was defined as staining positivity higher than that of normal

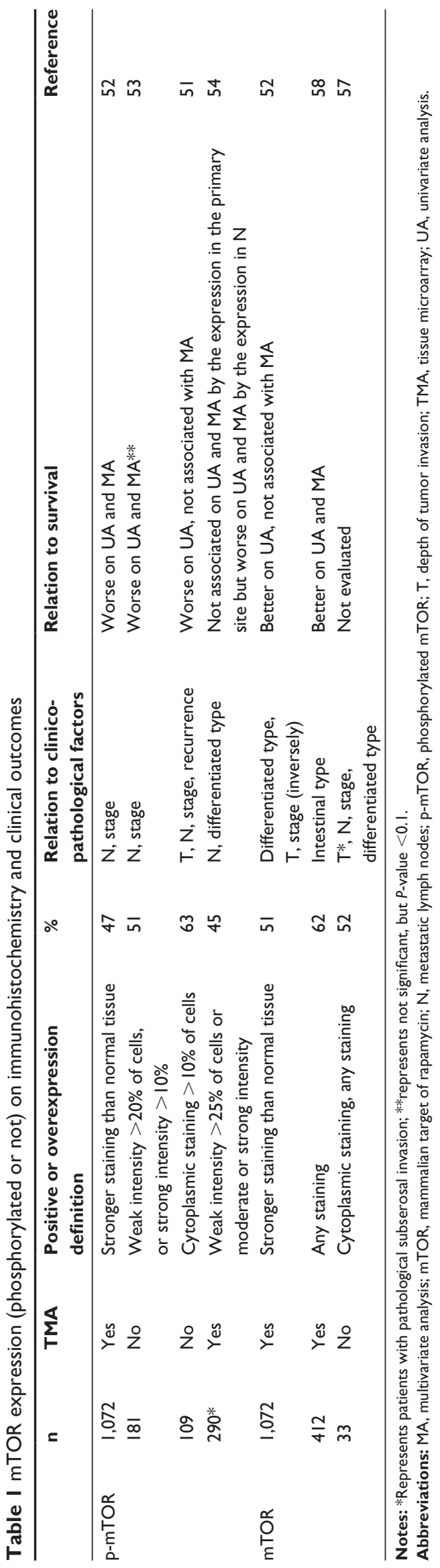


tissue in that study. Similar results were also obtained in another study, which reported that p-mTOR was detected mainly in cytoplasm and partly in cell membrane. ${ }^{53}$ In that study, positive staining was based on a scoring system in which staining intensity was multiplied by the percentage of positive tumor cells. An et al showed that p-mTOR expression on IHC analysis was associated with the extent of lymph-node metastasis in GC with subserosal invasion; moreover, p-mTOR expression in metastatic lymph nodes correlated with poor disease-free survival. ${ }^{54}$ Positive status of p-mTOR in cytoplasm or cell membrane was defined according to a scoring system based on staining intensity and extensity in that study. Lang et al reported that positive staining for p-mTOR was predominantly found at the tumor invasive front in $\mathrm{GC}$, whereas the adjacent normal gastric mucosa mainly stained negative for $\mathrm{p}-\mathrm{mTOR} .{ }^{55}$ Feng et al demonstrated cytoplasmic and membranous p-mTOR expression in GC, and positive p-mTOR expression was found even in chronic gastritis and intestinal metaplasia, which are considered precarcinomatous conditions. ${ }^{56}$

Nonactivated mTOR expression significantly correlated with lymph-node metastasis and pathological stage in GC. ${ }^{57}$ However, conflicting results have been obtained in other larger studies. mTOR expression was significantly associated with favorable survival in one study. ${ }^{58}$ Another study similarly reported that mTOR expression was more frequently detected in early-stage than in advanced-stage disease and was significantly associated with favorable survival. ${ }^{53}$ In addition, nuclear expression of phosphorylated S6K1, which is activated by $\mathrm{p}$-mTOR and participates in promotion of tumor progression, was associated with favorable outcomes in another study. ${ }^{58}$ These inconsistent results suggest that localization of S6K1 might have an inhibitory role in tumor progression, but this remains to be confirmed.

\section{Preclinical studies of $\mathbf{m T O R}$ inhibition in GC cells}

The downstream targets of mTOR, such as phosphorylated S6K1 and phosphorylated 4E-BP1, were decreased by mTOR inhibitors such as rapamycin or everolimus (RAD001) in some GC cell lines. ${ }^{59-63}$ Everolimus reduced tumor vascularization and cell proliferation independently of signal transducer and activator of transcription 3 , another downstream target of RTKs, in a mouse model. ${ }^{64}$ Everolimus, a specific mTORC1 inhibitor, significantly reduced peritoneal dissemination in a xenograft model. ${ }^{65}$ However, the basal phosphorylation level of 4E-BP1 was associated with sensitivity to everolimus in GC cell lines and a xenograft model. ${ }^{66} \mathrm{In}$ addition, rapamycin upregulated insulin-like growth factor-1 receptor expression in a $\mathrm{GC}$ cell line, and rictor indeed played an important role in that mechanism. ${ }^{60} \mathrm{~A}$ combination of rapamycin or everolimus with 5-fluorouracil displayed synergistic growth-inhibitory activity and downregulated thymidylate synthase in some GC cell lines. ${ }^{63,67,68}$ In addition, inhibition of mTOR is a key molecular event in enhancing fluorouracil-induced apoptosis in some HER2-amplified GC cell lines, regardless of sensitivity to trastuzumab ${ }^{69}$ Inhibition of mTOR by everolimus counteracted the effects of VEGF induction by sunitinib, significantly reducing tumor in a GC xenograft model. ${ }^{62}$ Interestingly, Ji et al demonstrated that everolimus and an Akt inhibitor induced beclin-1 expression and activated autophagic cell death pathway by extracellular signal-regulated kinases. ${ }^{70}$

\section{Critical trials of $\mathrm{mTOR}$-targeted treatment in GC}

Everolimus has been investigated in several clinical trials of $\mathrm{mGC}$, and the critical trials are summarized in Table 2. In a Phase I dose-escalation $(2.5,5$, or $10 \mathrm{mg} /$ day $)$ study of everolimus alone in nine patients with advanced solid tumors, including GC, dose-limiting toxicity (DLT) occurred, and the maximum-tolerated dose and recommended dose (RD) were determined to be $10 \mathrm{mg} /$ day. One patient with GC markedly responded to $10 \mathrm{mg} /$ day of everolimus. ${ }^{71} \mathrm{In}$ patients with esophagogastric cancer who had previously received chemotherapy, a Phase I dose-escalation study of everolimus $(5,7.5$, or $10 \mathrm{mg}$ /day) plus intravenous mitomycin C (5 mg/m $\mathrm{m}^{2}$, every 3 weeks) reported one case of DLT at $10 \mathrm{mg} / \mathrm{day}$ in the extended portion of the trial. The maximum-tolerated dose and RD of everolimus were therefore estimated to be $10 \mathrm{mg} /$ day. The most frequent grade 3 or higher adverse events were leukopenia (19\%) and neutropenia (19\%). Three patients (19\%) had a partial response, and four $(25 \%)$ had stable disease. Antitumor activity was highest in the $10 \mathrm{mg} /$ day cohort. ${ }^{72}$

In a Phase II trial in Japan, everolimus (10 mg/day) was administered to 53 patients with $\mathrm{mGC}$ previously treated by chemotherapy. ${ }^{73}$ The disease control rate, the endpoint of that study, was $56 \%$, although all of these patients had stable disease, with no complete or partial response to treatment. The MST was 10.1 months, and the median PFS was 2.7 months. These results suggested that the primary benefit of everolimus was disease stabilization in mGC. Twenty-four patients $(45 \%)$ had grade 3 or 4 adverse events, including anemia (9.4\%), hyponatremia (9.4\%), elevated gamma-glutamyltransferase levels $(7.5 \%)$, and lymphopenia $(7.5 \%)$. 
Table 2 Clinical trials of everolimus against metastatic gastric cancer

\begin{tabular}{|c|c|c|c|c|c|c|}
\hline Phase & Treatment arm & Chemotherapy line & $\mathbf{n}$ & Main endpoint & Results & Reference \\
\hline I & Everolimus & 2nd or more & 9 & MTD & RD and MTD was $10 \mathrm{mg} /$ day & 71 \\
\hline I & Everolimus + mitomycin $\mathrm{C}$ & 2nd or more & 16 & MTD & RD and MTD of everolimus was $10 \mathrm{mg} /$ day & 72 \\
\hline I & Everolimus + capecitabine & 3rd or more & 15 & MTD & RD and MTD of everolimus was $10 \mathrm{mg} /$ day & 75 \\
\hline \multirow[t]{2}{*}{ II } & Everolimus & 2nd, 3rd & 53 & DCR & DCR $56 \%$ (no CR/PR) & 73 \\
\hline & & & & & Median OS 10.1 M, median PFS $2.7 \mathrm{M}$ & \\
\hline \multirow[t]{2}{*}{ II } & Everolimus & 2nd & 54 & ORR/DCR & ORR 3.9\% (no CR), DCR 39\% & 74 \\
\hline & & & & & Median OS 8.3 M, median PFS I.7 M & \\
\hline \multirow[t]{2}{*}{ II } & Everolimus + capecitabine & $3 r d$ or more & 47 & ORR & ORR II\% (no CR), DCR 49\% & 76 \\
\hline & & & & & Median OS 4.9 M, median PFS $2.6 \mathrm{M}$ & \\
\hline \multirow[t]{3}{*}{ III } & Everolimus versus placebo & 2nd, 3rd & 656 & OS & ORR $4.5 \%$ versus $2.1 \%$ & 77 \\
\hline & (GRANITE-I) & & & & Median OS $5.4 \mathrm{M}$ versus $4.3 \mathrm{M}$ & \\
\hline & & & & & Median PFS I.7 M versus I.4 M & \\
\hline
\end{tabular}

Abbreviations: CR, complete response; DCR, disease control rate; M, months; MTD, maximum-tolerated dose; ORR, overall response rate; OS, overall survival; PFS, progression-free survival; $P R$, partial response; RD, recommended dose.

In a Phase II trial performed in Korea, everolimus $(10 \mathrm{mg} /$ day) was administered to 44 patients who had $\mathrm{mGC}$ with no response to fluoropyrimidine or platinum therapy. ${ }^{74}$ Two patients (3.7\%) had a partial response, and the disease control rate was $39 \%$. The MST was 8.3 months, and the median PFS was 1.7 months. In that study, the expressions of p-mTOR, S6K1, and 4E-BP1 were evaluated on IHC analysis as biomarkers. $\mathrm{pS}^{\mathrm{Ser} 240 / 4}$ was suggested to be a predictive biomarker because it was significantly associated with worse PFS and was moderately associated with overall survival.

A combination of everolimus plus capecitabine was given as third-line or subsequent chemotherapy to patients with mGC in a Korean study ${ }^{75}$ In this Phase I study, the RDs of everolimus and capecitabine were respectively estimated to be $10 \mathrm{mg}$ /day and $1,300 \mathrm{mg}$ /day (on days $1-14$, every 3 weeks), although multiple DLT associated with grade 3 adverse effects, including hyperglycemia, hyponatremia, stomatitis, thrombocytopenia, and hypophosphatemia, occurred at the upper dose level. In a Phase II study of 47 patients with $\mathrm{mGC}$, including 43 patients assessable for treatment response, five patients (11\%) had a partial response, and $18(38 \%)$ had stable disease. ${ }^{76}$ The MST and median PFS were 4.9 and 2.6 months, respectively.

However, a Phase III study (GRANITE-1) of everolimus resulted in unfavorable outcomes as second- or third-line therapy. ${ }^{77}$ Six hundred fifty-six patients with $\mathrm{mGC}$ were randomly assigned to everolimus $10 \mathrm{mg}$ /day or to matching placebo (assignment ratio: 2:1), both given with best supportive care. MST was 5.4 months with everolimus and 4.3 months with placebo $(P=0.124)$ although median PFS was 1.7 months and 1.4 months in the everolimus group and placebo group, respectively $(P<0.001)$.

\section{Clinical trials of mTOR-targeted treatment in various cancers}

The clinical efficacy of everolimus, an mTOR inhibitor, was initially demonstrated in a worldwide Phase III study in patients with renal cell carcinoma. ${ }^{78}$ In that study, patients with metastatic renal cell carcinoma who had progressive disease while receiving sunitinib, sorafenib, or both were randomly assigned in a two-to-one ratio to receive everolimus (10 mg/day) or placebo in conjunction with best supportive care. Treatment with everolimus prolonged PFS as compared with placebo (median PFS 4.0 versus 1.9 months, $P<0.0001$ ). Adverse events of grade 3 or 4 were significantly more frequent in the everolimus group and included stomatitis (3\%) and hypercholesterolemia (12\%).

Another mTOR inhibitor, ridaforolimus, was effective against sarcoma in a Phase III study. ${ }^{79}$ Patients with metastatic soft tissue or bone sarcomas who had an objective response or stable disease during prior chemotherapy were randomly assigned to receive ridaforolimus (40 mg for 5 days every week) or placebo. Ridaforolimus treatment significantly improved PFS as compared with placebo (4.1 versus 3.4 months, $P=0.001$ ) while the MST in the ridaforolimus and placebo groups was 21.1 and 19.9 months, respectively $(P=0.46)$.

However, disappointing results of monotherapy with mTOR-targeted agents have been reported in several types of cancers despite feasible adverse effects. In a Phase II study of everolimus $(10 \mathrm{mg} /$ day) in patients with gemcitabine-refractory metastatic pancreatic cancer, no response was noted, and only $21 \%$ of patients had stable disease. ${ }^{80}$ The MST and median PFS were 4.5 and 1.8 months, respectively. In other Phase II studies of temsirolimus ( $25 \mathrm{mg} /$ week) or everolimus ( $30 \mathrm{mg} /$ week) in pancreatic cancer, most of the patients given either of these 
mTOR inhibitors had received prior chemotherapy. ${ }^{81}$ The study of temsirolimus was terminated because of clinically significant adverse effects. Everolimus produced no response or disease stability, with an MST of 2.9 months and a median PFS of 1.6 months in that study.

In small-cell lung cancer, everolimus $(10 \mathrm{mg} /$ day $)$ had limited antitumor activity in previously treated patients, with an MST of 6.7 months and a median PFS of 1.3 months. ${ }^{82} \mathrm{In}$ that study, high phosphorylated Akt expression was modestly associated with overall survival, and baseline S6K1 expression was significantly higher in patients with disease control than in those with progression.

In a Phase II study of everolimus, unsatisfactory results were obtained in patients with hepatocellular carcinoma, most of whom had received prior chemotherapy. ${ }^{83}$ The MST and median PFS were 8.4 and 3.8 months, respectively.

\section{Discussion}

In Phase II clinical trials, a response to everolimus alone was rare among patients with $\mathrm{mGC}$, although everolimus appeared to slightly prolong survival. However, a Phase III study (GRANITE-1) showed no efficacy of everolimus in terms of overall survival as compared with a placebo. Everolimus thus might not be effective as a single agent for mGC. As secondline monotherapy for gastric cancer, better responses to other cytotoxic agents, such as paclitaxel and irinotecan, have been obtained as compared with everolimus. In one Phase III study the response rate was $20.9 \%$ for paclitaxel and $13.6 \%$ for irinotecan ${ }^{84}$ Poor response to everolimus alone has been demonstrated in various malignancies; thus, everolimus was expected to maintain stable disease in Phase III studies. Even in a Phase III study that obtained favorable outcomes in renal cell carcinoma, the response rate was only $3 \%{ }^{78}$ Poor response rates may be related to incomplete inhibition of the Akt/mTOR signaling pathway. In some preclinical studies, everolimus can incompletely inhibit the mTOR signaling pathway and suppress tumor cell proliferation by activation of insulin-like growth factor-1 receptor. ${ }^{60,70}$

However, adding everolimus to a standard regimen has not yet been shown to be beneficial. Combined chemotherapy with everolimus and a cytotoxic agent may improve the response rate as well as overall survival. In another Phase III study (AIO-STO-0111) of second- or third-line chemotherapy for gastric cancer, the effectiveness and safety of combined chemotherapy with everolimus plus paclitaxel are being compared with those of a placebo plus paclitaxel.$^{85}$ As mentioned above, clinical trials combining everolimus with capecitabine have obtained promising results in patients with mGC refractory to chemotherapy. ${ }^{74}$ In several studies of GC cells, combining an mTOR inhibitor with fluorouracil, another TKI, or an Akt inhibitor boosted antitumor activity. ${ }^{67-70}$

Everolimus has not been evaluated as first-line chemotherapy for mGC. In metastatic renal cell carcinoma, a randomized clinical trial was performed to compare everolimus with sunitinib as first-line chemotherapy. Unfortunately, patients who received everolimus had shorter PFS than those who received sunitinib. ${ }^{86}$ The efficacy of everolimus as first-line monotherapy thus appears to be limited; therefore, combining everolimus with cytotoxic agents or other targeting agents is also needed for first-line chemotherapy.

mTOR inhibitors have been administered to unselected patients with mGC in clinical trials. Enhancement of effectiveness requires that mTOR inhibitors are given to patients whose tumors are regulated by mTOR-dependent signaling pathways. The tumor expression of S6K1 might be a potential predictor of the response to everolimus, although small clinical trials performed to date have yielded conflicting results. The aforementioned Phase III studies (GRANITE-1 and AIO-STO-0111) include exploratory biomarker research designed to evaluate the predictive value of the expression or mutation of molecules related to the Akt/mTOR signaling pathway. These biomarker studies may lead to the realization of targeted therapy for selected patients with mGC in the future.

\section{Disclosure}

The authors report no conflicts of interest in this work.

\section{References}

1. Jemal A, Bray F, Center MM, Ferlay J, Ward E, Forman D. Global cancer statistics. CA Cancer J Clin. 2011;61(2):69-90.

2. Catalano V, Labianca R, Beretta GD, Gatta G, de Braud F, Van Cutsem E. Gastric cancer. Crit Rev Oncol Hematol. 2009;71(2):127-164.

3. Nashimoto A, Akazawa K, Isobe Y, et al. Gastric cancer treated in 2002 in Japan: 2009 annual report of the JGCA nationwide registry. Gastric Cancer. 2013;16(1):1-27.

4. Koizumi W, Narahara H, Hara T, et al. S-1 plus cisplatin versus S-1 alone for first-line treatment of advanced gastric cancer (SPIRITS trial): a phase III trial. Lancet Oncol. 2008;9(3):215-221.

5. Narahara H, Iishi H, Imamura H, et al. Randomized phase III study comparing the efficacy and safety of irinotecan plus S-1 with S-1 alone as first-line treatment for advanced gastric cancer (study GC0301/ TOP-002). Gastric Cancer. 2011;14(1):72-80.

6. Koizumi W, Kim YH, Fujii M, et al; The JACCRO and KCSG Study Group. Addition of docetaxel to S-1 without platinum prolongs survival of patients with advanced gastric cancer: a randomized study (START). J Cancer Res Clin Oncol. 2014;140(2):319-328.

7. Ajani JA, Rodriguez W, Bodoky G, et al. Multicenter phase III comparison of cisplatin/S-1 with cisplatin/infusional fluorouracil in advanced gastric or gastroesophageal adenocarcinoma study: the FLAGS trial. J Clin Oncol. 2010;28(9):1547-1553. 
8. Ajani JA, Faust J, Ikeda K, et al. Phase I pharmacokinetic study of S-1 plus cisplatin in patients with advanced gastric carcinoma. J Clin Oncol. 2005;23(28):6957-6965.

9. Cunningham D, Starling N, Rao S, et al; Upper Gastrointestinal Clinical Studies Group of the National Cancer Research Institute of the United Kingdom. Capecitabine and oxaliplatin for advanced esophagogastric cancer. $N$ Engl J Med. 2008;358(1):36-46.

10. Van Cutsem E, Moiseyenko VM, Tjulandin S, et al. Phase III study of docetaxel and cisplatin plus fluorouracil compared with cisplatin and fluorouracil as first-line therapy for advanced gastric cancer: a report of the V325 Study Group. J Clin Oncol. 2006;24(31):4991-4997.

11. Koizumi W, Nakayama N, Tanabe S, et al. A multicenter phase II study of combined chemotherapy with docetaxel, cisplatin, and S-1 in patients with unresectable or recurrent gastric cancer (KDOG 0601). Cancer Chemother Pharmacol. 2012;69(2):407-413.

12. Takeuchi K, Ito F. Receptor tyrosine kinases and targeted cancer therapeutics. Biol Pharm Bull. 2011;34(12):1774-1780.

13. Kim MA, Lee HS, Lee HE, Jeon YK, Yang HK, Kim WH. EGFR in gastric carcinomas: prognostic significance of protein overexpression and high gene copy number. Histopathology. 2008;52(6):738-746.

14. Begnami MD, Fukuda E, Fregnani JH, et al. Prognostic implications of altered human epidermal growth factor receptors (HERs) in gastric carcinomas: HER 2 and HER3 are predictors of poor outcome. $J$ Clin Oncol. 2011;29(22):3030-3036.

15. Jørgensen JT, Hersom M. HER2 as a prognostic marker in gastric cancer - a systematic analysis of data from the literature. $J$ Cancer. 2012;3:137-144.

16. Hirashima Y, Yamada Y, Matsubara J, et al. Impact of vascular endothelial growth factor receptor 1, 2, and 3 expression on the outcome of patients with gastric cancer. Cancer Sci. 2009;100(2):310-315.

17. Mimori K, Fukagawa T, Kosaka Y, et al. Hematogenous metastasis in gastric cancer requires isolated tumor cells and expression of vascular endothelial growth factor receptor-1. Clin Cancer Res. 2008;14(9):2609-2616.

18. Ha SY, Lee J, Kang SY, et al. MET overexpression assessed by new interpretation method predicts gene amplification and poor survival in advanced gastric carcinomas. Mod Pathol. 2013;26(12):1632-1641.

19. Lee HE, Kim MA, Lee HS, et al. MET in gastric carcinomas: comparison between protein expression and gene copy number and impact on clinical outcome. Br J Cancer. 2012;107(2):325-333.

20. Bang YJ, Van Cutsem E, Feyereislova A, et al; ToGA Trial Investigators. Trastuzumab in combination with chemotherapy versus chemotherapy alone for treatment of HER2-positive advanced gastric or gastro-oesophageal junction cancer (ToGA): a phase 3, open-label, randomised controlled trial. Lancet. 2010;376(9742):687-697.

21. Lordick F, Kang YK, Chung HC, et al; Arbeitsgemeinschaft Internistische Onkologie and EXPAND Investigators. Capecitabine and cisplatin with or without cetuximab for patients with previously untreated advanced gastric cancer (EXPAND): a randomised, open-label phase 3 trial. Lancet Oncol. 2013;14(6):490-499.

22. Waddell T, Chau I, Cunningham D, et al. Epirubicin, oxaliplatin, and capecitabine with or without panitumumab for patients with previously untreated advanced oesophagogastric cancer (REAL3): a randomised, open-label phase 3 trial. Lancet Oncol. 2013;14(6):481-489.

23. Bang YJ. A randomized, open-label, phase III study of lapatinib in combination with weekly paclitaxel versus weekly paclitaxel alone in the second-line treatment of HER2 amplified advanced gastric cancer in Asian populations: Tytan study. J Clin Oncol. 2013;31(Suppl 4): abstract 11 .

24. Ohtsu A, Shah MA, Van Cutsem E, et al. Bevacizumab in combination with chemotherapy as first-line therapy in advanced gastric cancer: a randomized, double-blind, placebo-controlled phase III study. J Clin Oncol. 2011;29(30):3968-3976.

25. Van Cutsem E, de Haas S, Kang YK, et al. Bevacizumab in combination with chemotherapy as first-line therapy in advanced gastric cancer: a biomarker evaluation from the AVAGAST randomized phase III trial. J Clin Oncol. 2012;30(17):2119-2127.
26. Fuchs CS, Tomasek J, Yong CJ, et al; REGARD Trial Investigators. Ramucirumab monotherapy for previously treated advanced gastric or gastro-oesophageal junction adenocarcinoma (REGARD): an international, randomised, multicentre, placebo-controlled, phase 3 trial. Lancet. 2014;383(9911):31-39.

27. Iveson T, Donehower RC, Davidenko I, et al. 6504 ORAL safety and efficacy of epirubicin, cisplatin, and capecitabine plus rilotumumab as first-line treatment for unresectable locally advanced or metastatic gastric or esophagogastric junction adenocarcinoma. Eur J Cancer. 2011;47(Suppl 1):S443.

28. Shah MA, Wainberg ZA, Catenacci DV, et al. Phase II study evaluating 2 dosing schedules of oral foretinib (GSK1363089), cMET/ VEGFR2 inhibitor, in patients with metastatic gastric cancer. PLoS One. 2013;8(3):e54014.

29. Sun W, Powell M, O'Dwyer PJ, Catalano P, Ansari RH, Benson AB. Phase II study of sorafenib in combination with docetaxel and cisplatin in the treatment of metastatic or advanced gastric and gastroesophageal junction adenocarcinoma: ECOG 5203. J Clin Oncol. 2010;28(18): 2947-2951.

30. Bang YJ, Kang YK, Kang WK, et al. Phase II study of sunitinib as second-line treatment for advanced gastric cancer. Invest New Drugs. 2011;29(6):1449-1458.

31. Moehler M, Mueller A, Hartmann JT, et al; German Arbeitsgemeinschaft Internistische Onkologie (AIO). An open-label, multicentre biomarker-oriented AIO phase II trial of sunitinib for patients with chemo-refractory advanced gastric cancer. Eur J Cancer. 2011;47(10): $1511-1520$

32. Fingar DC, Richardson CJ, Tee AR, Cheatham L, Tsou C, Blenis J. mTOR controls cell cycle progression through its cell growth effectors S6K1 and 4E-BP1/eukaryotic translation initiation factor 4E. Mol Cell Biol. 2004;24(1):200-216.

33. Edinger AL, Thompson $\mathrm{CB}$. Akt maintains cell size and survival by increasing mTOR-dependent nutrient uptake. Mol Biol Cell. 2002;13(7):2276-2288.

34. Al-Batran SE, Ducreux M, Ohtsu A. mTOR as a therapeutic target in patients with gastric cancer. Int J Cancer. 2012;130(3):491-496.

35. Hay N, Sonenberg N. Upstream and downstream of mTOR. Genes Dev. 2004;18(16):1926-1945.

36. Su B, Jacinto E. Mammalian TOR signaling to the AGC kinases. Crit Rev Biochem Mol Biol. 2011;46(6):527-547.

37. Hara K, Maruki Y, Long X, et al. Raptor, a binding partner of target of rapamycin (TOR), mediates TOR action. Cell. 2002;110(2): 177-189.

38. Loewith R, Jacinto E, Wullschleger S, et al. Two TOR complexes, only one of which is rapamycin sensitive, have distinct roles in cell growth control. Mol Cell. 2002;10(3):457-468.

39. Kim DH, Sarbassov DD, Ali SM, et al. mTOR interacts with raptor to form a nutrient-sensitive complex that signals to the cell growth machinery. Cell. 2002;110(2):163-175.

40. Wang L, Lawrence JC Jr, Sturgill TW, Harris TE. Mammalian target of rapamycin complex 1 (mTORC1) activity is associated with phosphorylation of raptor by mTOR. J Biol Chem. 2009;284(22):14693-14697.

41. Foster KG, Acosta-Jaquez HA, Romeo Y, et al. Regulation of mTOR complex 1 (mTORC1) by raptor Ser863 and multisite phosphorylation. J Biol Chem. 2010;285(1):80-94.

42. Janus A, Robak T, Smolewski P. The mammalian target of the rapamycin (mTOR) kinase pathway: its role in tumourigenesis and targeted antitumour therapy. Cell Mol Biol Lett. 2005;10(3):479-498.

43. Sabatini DM. mTOR and cancer: insights into a complex relationship. Nat Rev Cancer. 2006;6(9):729-734.

44. Sarbassov DD, Ali SM, Kim DH, et al. Rictor, a novel binding partner of mTOR, defines a rapamycin-insensitive and raptor-independent pathway that regulates the cytoskeleton. Curr Biol. 2004;14(14): 1296-1302.

45. Jacinto E, Loewith R, Schmidt A, et al. Mammalian TOR complex 2 controls the actin cytoskeleton and is rapamycin insensitive. Nat Cell Biol. 2004;6(11):1122-1128. 
46. Wander SA, Hennessy BT, Slingerland JM. Next-generation mTOR inhibitors in clinical oncology: how pathway complexity informs therapeutic strategy. J Clin Invest. 2011;121(4):1231-1241.

47. Dowling RJ, Topisirovic I, Fonseca BD, Sonenberg N. Dissecting the role of mTOR: lessons from mTOR inhibitors. Biochim Biophys Acta. 2010;1804(3):433-439.

48. Lorin S, Hamaï A, Mehrpour M, et al. Autophagy regulation and its role in cancer. Semin Cancer Biol. 2013;23(5):361-379.

49. Bachmann RA, Kim JH, Wu AL, Park IH, Chen J. A nuclear transport signal in mammalian target of rapamycin is critical for its cytoplasmic signaling to S6 kinase 1.J Biol Chem. 2006;281(11):7357-7363.

50. Rosner M, Hengstschläger M. Cytoplasmic and nuclear distribution of the protein complexes $\mathrm{mTORC} 1$ and $\mathrm{mTORC} 2$ : rapamycin triggers dephosphorylation and delocalization of the mTORC2 components rictor and sin1. Hum Mol Genet. 2008;17(19):2934-2948.

51. Murayama T, Inokuchi M, Takagi $Y$, et al. Relation between outcomes and localisation of p-mTOR expression in gastric cancer. Br J Cancer. 2009;100(5):782-788.

52. Yu G, Wang J, Chen Y, et al. Overexpression of phosphorylated mammalian target of rapamycin predicts lymph node metastasis and prognosis of chinese patients with gastric cancer. Clin Cancer Res. 2009;15(5):1821-1829.

53. Xu DZ, Geng QR, Tian Y, et al. Activated mammalian target of rapamycin is a potential therapeutic target in gastric cancer. BMC Cancer. 2010;10:536.

54. An JY, Kim KM, Choi MG, et al. Prognostic role of p-mTOR expression in cancer tissues and metastatic lymph nodes in pT2b gastric cancer. Int J Cancer. 2010;126(12):2904-2913.

55. Lang SA, Gaumann A, Koehl GE, et al. Mammalian target of rapamycin is activated in human gastric cancer and serves as a target for therapy in an experimental model. Int J Cancer. 2007;120(8):1803-1810.

56. Feng W, Brown RE, Trung CD, et al. Morphoproteomic profile of mTOR, Ras/Raf kinase/ERK, and NF-kappaB pathways in human gastric adenocarcinoma. Ann Clin Lab Sci. 2008;38(3):195-209.

57. Li M, Sun H, Song L, Gao X, Chang W, Qin X. Immunohistochemical expression of mTOR negatively correlates with PTEN expression in gastric carcinoma. Oncol Lett. 2012;4(6):1213-1218.

58. Xiao L, Wang YC, Li WS, Du Y. The role of mTOR and phosphop70S6K in pathogenesis and progression of gastric carcinomas: an immunohistochemical study on tissue microarray. J Exp Clin Cancer Res. 2009;28:152.

59. Hashimoto I, Koizumi K, Tatematsu M, et al. Blocking on the CXCR4/ mTOR signalling pathway induces the anti-metastatic properties and autophagic cell death in peritoneal disseminated gastric cancer cells. Eur J Cancer. 2008;44(7):1022-1029.

60. Lang SA, Hackl C, Moser C, et al. Implication of RICTOR in the mTOR inhibitor-mediated induction of insulin-like growth factor-I receptor (IGF-IR) and human epidermal growth factor receptor-2 (Her2) expression in gastrointestinal cancer cells. Biochim Biophys Acta. 2010;1803(4):435-442.

61. Cejka D, Preusser M, Woehrer A, et al. Everolimus (RAD001) and anti-angiogenic cyclophosphamide show long-term control of gastric cancer growth in vivo. Cancer Biol Ther. 2008;7(9): 1377-1385.

62. Fuereder T, Jaeger-Lansky A, Hoeflmayer D, et al. mTOR inhibition by everolimus counteracts VEGF induction by sunitinib and improves anti-tumor activity against gastric cancer in vivo. Cancer Lett. 2010;296(2):249-256.

63. Shigematsu H, Yoshida K, Sanada Y, et al. Rapamycin enhances chemotherapy-induced cytotoxicity by inhibiting the expressions of TS and ERK in gastric cancer cells. Int J Cancer. 2010;126(11):2716-2725.

64. Thiem S, Pierce TP, Palmieri M, et al. mTORC1 inhibition restricts inflammation-associated gastrointestinal tumorigenesis in mice. $J$ Clin Invest. 2013;123(2):767-781.

65. Taguchi F, Kodera Y, Katanasaka Y, Yanagihara K, Tamura T, Koizumi F. Efficacy of RAD001 (everolimus) against advanced gastric cancer with peritoneal dissemination. Invest New Drugs. 2011;29(6):1198-1205.
66. Nishi T, Iwasaki K, Ohashi N, et al. Phosphorylation of 4E-BP1 predicts sensitivity to everolimus in gastric cancer cells. Cancer Lett. 2013; 331(2):220-229.

67. Matsuzaki T, Yashiro M, Kaizaki R, et al. Synergistic antiproliferative effect of mTOR inhibitors in combination with 5-fluorouracil in scirrhous gastric cancer. Cancer Sci. 2009;100(12): 2402-2410.

68. Lee KH, Hur HS, Im SA, et al. RAD001 shows activity against gastric cancer cells and overcomes 5-FU resistance by downregulating thymidylate synthase. Cancer Lett. 2010;299(1):22-28.

69. Tomioka H, Mukohara T, Kataoka Y, et al. Inhibition of the mTOR/ S6K signal is necessary to enhance fluorouracil-induced apoptosis in gastric cancer cells with HER2 amplification. Int $J$ Oncol. 2012;41(2):551-558.

70. Ji D, Zhang Z, Cheng L, et al. The combination of RAD001 and MK-2206 exerts synergistic cytotoxic effects against PTEN mutant gastric cancer cells: involvement of MAPK-dependent autophagic, but not apoptotic cell death pathway PLoS One. 2014;9(1):e85116.

71. Okamoto I, Doi T, Ohtsu A, et al. Phase I clinical and pharmacokinetic study of RAD001 (everolimus) administered daily to Japanese patients with advanced solid tumors. Jpn J Clin Oncol. 2010;40(1):17-23.

72. Werner D, Atmaca A, Pauligk C, Pustowka A, Jäger E, Al-Batran SE. Phase I study of everolimus and mitomycin $\mathrm{C}$ for patients with metastatic esophagogastric adenocarcinoma. Cancer Med. 2013;2(3): 325-333.

73. Doi T, Muro K, Boku N, et al. Multicenter phase II study of everolimus in patients with previously treated metastatic gastric cancer. $J$ Clin Oncol. 2010;28(11):1904-1910.

74. Yoon DH, Ryu MH, Park YS, et al. Phase II study of everolimus with biomarker exploration in patients with advanced gastric cancer refractory to chemotherapy including fluoropyrimidine and platinum. $\mathrm{Br} J$ Cancer. 2012;106(6):1039-1044.

75. Lim T, Lee J, Lee DJ, et al. Phase I trial of capecitabine plus everolimus (RAD001) in patients with previously treated metastatic gastric cancer. Cancer Chemother Pharmacol. 2011;68(1):255-262.

76. Lee SJ, Lee J, Lee J, et al. Phase II trial of capecitabine and everolimus (RAD001) combination in refractory gastric cancer patients. Invest New Drugs. 2013;31(6):1580-1586.

77. Ohtsu A, Ajani JA, Bai YX, et al. Everolimus for previously treated advanced gastric cancer: results of the randomized, double-blind, phase III GRANITE-1 study. J Clin Oncol. 2013;31(31):3935-3943.

78. Motzer RJ, Escudier B, Oudard S, et al; RECORD-1 Study Group. Efficacy of everolimus in advanced renal cell carcinoma: a doubleblind, randomised, placebo-controlled phase III trial. Lancet 2008;372(9637):449-456.

79. Demetri GD, Chawla SP, Ray-Coquard I, et al. Results of an international randomized phase III trial of the mammalian target of rapamycin inhibitor ridaforolimus versus placebo to control metastatic sarcomas in patients after benefit from prior chemotherapy. J Clin Oncol. 2013;31(19):2485-2492.

80. Wolpin BM, Hezel AF, Abrams T, et al. Oral mTOR inhibitor everolimus in patients with gemcitabine-refractory metastatic pancreatic cancer. J Clin Oncol. 2009;27(2):193-198.

81. Javle MM, Shroff RT, Xiong H, et al. Inhibition of the mammalian target of rapamycin (mTOR) in advanced pancreatic cancer: results of two phase II studies. BMC Cancer. 2010;10:368.

82. Tarhini A, Kotsakis A, Gooding W, et al. Phase II study of everolimus (RAD001) in previously treated small cell lung cancer. Clin Cancer Res. 2010;16(23):5900-5907.

83. Zhu AX, Abrams TA, Miksad R, et al. Phase 1/2 study of everolimus in advanced hepatocellular carcinoma. Cancer. 2011;117(22): 5094-5102.

84. Hironaka S, Ueda S, Yasui H, et al. Randomized, open-label, phase III study comparing irinotecan with paclitaxel in patients with advanced gastric cancer without severe peritoneal metastasis after failure of prior combination chemotherapy using fluoropyrimidine plus platinum: WJOG 4007 trial. J Clin Oncol. 2013;31(35):4438-4444. 
85. A Randomized, Double Blind Study Evaluating Paclitaxel With and Without RAD001 in Patients With Gastric Carcinoma After Prior Chemotherapy (AIO-STO-0111). Avaliable from: http://clinicaltrials. gov/show/NCT01248403.

86. Motzer RJ, Nosov D, Eisen T, et al. Record-3: Phase II randomized trial comparing sequential first-line everolimus (EVE) and secondline sunitinib (SUN) versus first-line SUN and second-line EVE in patients with metastatic renal cell carcinoma (mRCC). J Clin Oncol. 2013;31(Suppl):abstract 4504.

\section{Publish your work in this journal}

Gastrointestinal Cancer: Targets and Therapy is an international, peer-reviewed, open access journal focusing on gastro-intestinal cancer research, identification of therapeutic targets and the optimal use of preventative and integrated treatment interventions to achieve improved outcomes, enhanced survival and quality of life for the

Submit your manuscript here: http://www.dovepress.com/gastro-intestinal-cancer-targets-and-therapy-journal cancer patient. The manuscript management system is completely online and includes a very quick and fair peer-review system. Visit http://www.dovepress.com/testimonials.php to read real quotes from published authors. 